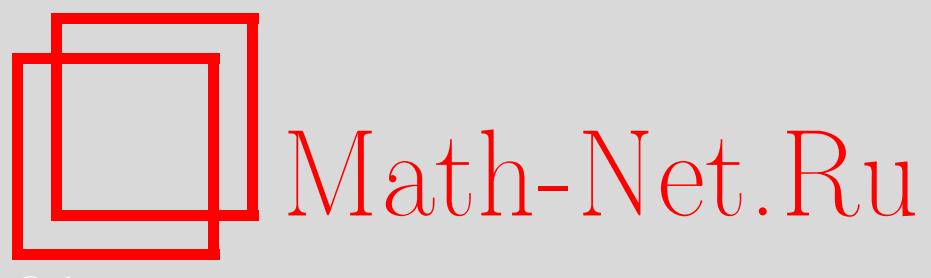

H. С. Романовский, Теорема Гильберта о нулях (Nullstellensatz) в алгебраической геометрии над жесткими разрешимыми группами, Изв. РАН. Сер. матем., 2015, том 79, выпуск 5, 201-214

DOI: https://doi.org/10.4213/im8295

Использование Общероссийского математического портала Math-Net.Ru подразумевает, что вы прочитали и согласны с пользовательским соглашением http: //www. mathnet.ru/rus/agreement

Параметры загрузки:

IP : 52.6 .47 .48

26 апреля 2023 г., 02:47:54

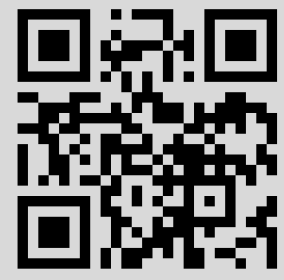




\title{
Н. С. Романовский \\ Теорема Гильберта о нулях (Nullstellensatz) в алгебраической геометрии над жесткими разрешимыми группами
}

\begin{abstract}
Обсуждается вопрос о том, какой вид может иметь аналог классической теоремы Гильберта о нулях (Nullstellensatz) в алгебраической геометрии над достаточно хорошим классом групп, и доказывается такой аналог в классе жестких разрешимых групп.

Библиография: 8 наименований.
\end{abstract}

Ключевые слова: группа, разрешимая, алгебраическая геометрия.

DOI: $10.4213 / \operatorname{im} 8295$

\section{§ 1. Введение и постановка задачи}

Классическая теорема Гильберта о нулях состоит в том, что если $K$ - алгебраически замкнутое поле и имеется система полиномиальных над $K$ уравнений $\left\{f_{i}\left(x_{1}, \ldots, x_{n}\right)=0 \mid i \in I\right\}$, то уравнение $f\left(x_{1}, \ldots, x_{n}\right)=0$ является ее логическим следствием (выполняется на всех решениях системы в $K^{n}$ ) в том и только в том случае, когда многочлен $f$ в некоторой ненулевой степени принадлежит идеалу $\left(f_{i} \mid i \in I\right)$ кольца $K\left[x_{1}, \ldots, x_{n}\right]$. Можно сказать, что в этой теореме дан алгебраический способ построения всех логических следствий данной системы уравнений: $f$ получается из $f_{i}, i \in I$, при помощи операций сложения, вычитания, умножения на элементы кольца $K\left[x_{1}, \ldots, x_{n}\right]$ и извлечения корней. Слабая теорема Гильберта о нулях утверждает, что система имеет решение в $K^{n}$ тогда и только тогда, когда идеал $\left(f_{i} \mid i \in I\right)$ строго меньше всего кольца $K\left[x_{1}, \ldots, x_{n}\right]$, или, что равносильно, пересекается с $K$ по нулю.

В работах Г. Баумслага, А.Г. Мясникова и В.Н. Ремесленникова [1] и [2] были изложены основы алгебраической геометрии над группой. Мы напомним некоторые определения. Для данной группы $G$ назовем $G$-группой любую группу, содержащую $G$ в качестве фиксированной подгруппы. Пусть $F=G *\left\langle x_{1}, \ldots, x_{n}\right\rangle-$ свободное произведение группы $G$ и свободной группы с базой $\left\{x_{1}, \ldots, x_{n}\right\}$. Уравнением от $x=\left(x_{1}, \ldots, x_{n}\right)$ над $G$ называется выражение вида $v(x)=1$, где $v \in F$. Иногда под уравнением понимают левую часть, т. е. $v(x)$. Переменные $x_{i}$ принимают значения в $G$. Множество $S \subseteq G^{n}$ решений некоторой системы уравнений $\left\{v_{j}(x)=1 \mid j \in J\right\}$ называется алгебрачческим подмножеством в аффинном пространстве $G^{n}$.

Исследование выполнено за счет гранта Российского научного фонда (проект № 14-21-00065). 
Обозначим через $I(S)=\{v(x) \in F \mid v(s)=1, s \in S\}$ аннулятор непустого алгебраического множества $S$ и назовем координатной группой этого множества факторгруппу $\Gamma(S)=F / I(S)$. Очевидно, что $G$ вкладывается в эту факторгруппу и $\Gamma(S)$ как $G$-группа порождается образами элементов $x_{1}, \ldots, x_{n}$. Группу $F$ можно понимать как группу уравнений от $x$ с коэффициентами из $G$. В общем случае мы назовем группой уравнений от $x$ над $G$ любую группу $D$, которая порождается своей подгруппой $G$ и множеством элементов $\left\{x_{1}, \ldots, x_{n}\right\}$, если она удовлетворяет условию: всякое отображение $x \rightarrow\left(g_{1}, \ldots, g_{n}\right)$ определяет $G$-эпиморфизм $D \rightarrow G$. Понятно, что $D$ представляется в виде факторгруппы $F / H$. Среди таких $D$ существует группа $\Gamma\left(G^{n}\right)$ с максимальным $H=I\left(G^{n}\right)$. На пространстве $G^{n}$ определяется топология Зарисского: в качестве предбазы системы замкнутых множеств берутся все алгебраические множества. Доказано, что топология Зарисского на $G^{n}$ при любом $n$ является нётеровой тогда и только тогда, когда группа $G$ нётерова по уравнениям. Последнее означает, что любая система уравнений над $G$ от ограниченного числа переменных логически эквивалентна некоторой своей конечной подсистеме. Понятно, что трудно изучать алгебраическую геометрию над группой, если она не нётерова по уравнениям.

В работе Скотта [3] дано следующее определение алгебраически замкнутой группы: это такая группа $G$, что любая конечная система уравнений и неравенств над $G$, имеющая решение в некоторой $G$-группе, имеет решение и в $G$. В [3] также доказано, что всякая группа вкладывается в алгебраически замкнутую. Хотя это определение используется также в [1], на наш взгляд, правильно здесь употребить термин экзистенииально замкнутая группа, ибо по сути утверждается, что любая $\exists$-формула в сигнатуре теории групп с константами из $G$, истинная в некоторой $G$-группе, должна быть истинна в $G$. Как было показано в [1], для экзистенциально замкнутой группы $G$ имеет место теорема Гильберта о нулях в следующей простой формулировке: пусть $H \triangleleft F=G *\left\langle x_{1}, \ldots, x_{n}\right\rangle$ и $H$ порождается как нормальная подгруппа конечным числом элементов; тогда система $\left\{h\left(x_{1}, \ldots, x_{n}\right)=1 \mid h \in H\right\}$ имеет решение в $G^{n}$ в том и только в том случае, если $H \cap G=1$. Это также означает, что логические следствия конечной непротиворечивой системы уравнений составляют нормальную подгруппу группы $F$, порожденную этими уравнениями. Однако, по мнению автора, сформулированное утверждение не совсем верно называть аналогом классической теоремы Гильберта о нулях. Скорее, это аналог следующего простого по формулировке и доказательству утверждения: пусть $K-$ экзистенциально замкнутое коммутативное кольцо; тогда логические следствия конечной системы полиномиальных над $K$ уравнений $\left\{f_{i}\left(x_{1}, \ldots, x_{n}\right)=0 \mid i \in I\right\}$ в точности составляют идеал $\left(f_{i} \mid i \in I\right)$ кольца $K\left[x_{1}, \ldots, x_{n}\right]$ при условии, что этот идеал пересекается с $K$ по нулю.

Заметим, что любая экзистенциально замкнутая группа является "довольно плохой". Она, например, содержит в качестве подгрупп все конечные группы, а точнее, симметрическую группу $S_{\omega}=\bigcup_{n} S_{n}$. Нетрудно показать, что группа $S_{\omega}$ не является нётеровой по уравнениям, а значит, и сама группа $G$ 
не нётерова по уравнениям. Такую группу сложно использовать в алгебраической геометрии. Допустим теперь, что группа $G$ нётерова по уравнениям и для нее, как и выше, логические следствия конечной непротиворечивой системы уравнений в точности составляют нормальную подгруппу группы $F$, порожденную этими уравнениями. Возьмем тогда произвольную нормальную подгруппу $H$ из $F$, пересекающуюся с $G$ по единице, и рассмотрим систему уравнений $\left\{h\left(x_{1}, \ldots, x_{n}\right)=1 \mid h \in H\right\}$. Она эквивалентна некоторой своей конечной подсистеме, а тогда $H$ как нормальная подгруппа должна быть конечно порождена. Последнее при $n>1$ невозможно для любой группы $G$ в связи с тем, что в свободной группе $\left\langle x_{1}, \ldots, x_{n}\right\rangle$ не всякая нормальная подгруппа конечно порождена (как нормальная подгруппа). Здесь, разумеется, можно и необходимо изменить ситуацию в лучшую сторону следующим образом. Понятно, что все $G$-тождества, т. е. элементы из $I\left(G^{n}\right)$ являются логическими следствиями любой системы уравнений. Поэтому в качестве группы уравнений нужно рассматривать не $F$, а $\Gamma\left(G^{n}\right)=F / I\left(G^{n}\right)$. Тогда, например, если $G$ является конечно порожденной нильпотентной надабелевой группой, то и $\Gamma\left(G^{n}\right)$ будет конечно порожденной нильпотентной надабелевой группой, а значит, всякая ее нормальная подгруппа конечно порождена (как нормальная подгруппа). Однако в других интересных случаях этим свойством группа $\Gamma\left(G^{n}\right)$ не обладает, и поэтому логические следствия системы уравнений, вообще говоря, не будут получаться из исходных уравнений только с помощью стандартных операций перемножения, взятия обратного и сопряжения элементами группы. Нужно придумывать еще какие-то другие алгебраические правила вывода, если они вообще существуют.

Отметим, кроме того, что в классической алгебраической геометрии объединение конечного числа алгебраических множеств также является алгебраическим множеством, а значит, задается (конечной) системой уравнений. В алгебраической геометрии над нётеровыми по уравнениям группами замкнутое множество может не быть алгебраическим, но оно является объединением конечного числа неприводимых алгебраических множеств и поэтому задается (бескванторной) позитивной формулой - булевой комбинацией без отрицаний конечного множества уравнений. Поэтому в этом случае естественно рассматривать не системы уравнений, а позитивные формулы.

Теперь сформулируем, как можно трактовать теорему Гильберта о нулях в алгебраической геометрии над группами. Сначала ограничимся рассмотрением какого-то конкретного класса нётеровых по уравнениям групп, интересного с той или иной точки зрения. В этом классе должны существовать объекты, похожие на алгебраически замкнутые поля, назовем их алгебраически замкнутыми группами и докажем, что всякая группа рассматриваемого класса вкладывается в алгебраически замкнутую. Для данной алгебраически замкнутой группы $G$ и данной позитивной формулы от переменных $x_{1}, \ldots, x_{n}$, в которой равенства имеют вид $v=1, v \in \Gamma\left(G^{n}\right)$, следует указать алгебраические правила построения всех логических следствий (в виде позитивных формул). В этом и будет состоять теорема Гильберта о нулях в рассматриваемом классе групп. 
Было бы интересно, к примеру, найти подобный класс, содержащий свободные группы, и доказать для него теорему Гильберта о нулях. Причем постановка задачи будет проще, так как, скорее всего, для алгебраически замкнутого объекта будет выполняться условие $\Gamma\left(G^{n}\right)=F$ и вместо позитивных формул достаточно будет рассматривать обычные системы уравнений (напомним, что в топологии Зарисского над свободной группой всякое замкнутое множество является алгебраическим).

В настоящей работе поставленная выше задача решается для класса $\Sigma_{m}$ жестких групп (определение жесткой группы будет дано в следующем параграфе) ступени разрешимости не выше $m$ при любом натуральном $m$. Тем самым охватывается достаточно широкий спектр разрешимых групп, включающий в себя свободные разрешимые группы и итерированные сплетения абелевых групп без кручения, а также их подгруппы. Отметим, что в работе [4] был получен следующий принципиальный результат: всякая жесткая группа нётерова по уравнениям. В [5] определено понятие делимой жесткой группы и доказано, что произвольная $m$-жесткая группа вкладывается в делимую $m$-жесткую группу. В [6] были определены алгебраически замкнутые и экзистенциально замкнутые объекты в классе $\Sigma_{m}$ и установлено, что делимые $m$-жесткие группы в точности являются как теми, так и другими. Именно для этих групп мы указываем алгебраический способ построения всех логических следствий данной позитивной формулы и получаем информацию о неприводимых компонентах соответствующего замкнутого множества. В каком-то смысле формулировка основного утверждения близка к формулировке классической теоремы Гильберта о нулях. В последней говорится, что при выводе следствий кроме стандартных конструкций построения элементов идеала, порожденного данными уравнениями, нужно еще добавлять элементы $f$ такие, что $f^{k}$ для некоторого $0<k \in \mathbb{Z}$ лежит в этом идеале. В нашем случае стандартными являются конструкция построения нормальной подгруппы, порожденной данными уравнениями, и правила вывода исчисления высказываний, не содержащие отрицаний. K ним мы добавляем следующее новое правило. Пусть имеется уравнение $v=1$. Предположим, что элемент $v$ принадлежит подгруппе группы всех уравнений, которая распадается в полупрямое произведение $B A$, где $A$ - абелева нормальная подгруппа в $B A$ (на самом деле нами будут указаны конкретные такие подгруппы в конечном числе). Тогда $A$ можно рассматривать как правый модуль над групповым кольцом $\mathbb{Z} B$. Предположим, что $v$ имеет вид $a^{\gamma}$, где $a \in A, 0 \neq \gamma \in \mathbb{Z} B$. Понятно, что если для каких-то значений переменных $v=1$, то либо $a=1$, либо $\gamma=0$. Последнее равенство может выполняться лишь в том случае, если элемент $\gamma$ лежит в разностном идеале $(B-1) \mathbb{Z} B$. В этом случае представим $\gamma$ в виде $u_{1}+\cdots+u_{m}-v_{1}-\cdots-v_{m}$, где $u_{i}, v_{i} \in B$ и множества $\left\{u_{1}, \ldots, u_{m}\right\}$ и $\left\{v_{1}, \ldots, v_{m}\right\}$ не пересекаются. Это представление единственно с точностью до перестановки $u_{i}$ и $v_{i}$. Поставим в соответствие элементу $\gamma$ позитивную формулу

$$
\Theta(\gamma)=\bigvee_{\sigma \in S_{m}}\left(u_{1}=v_{\sigma(1)} \wedge \cdots \wedge u_{m}=v_{\sigma(m)}\right)
$$


Если $\gamma \notin(B-1) \mathbb{Z} B$, то считаем $\Theta(\gamma)$ ложью. Правило вывода состоит в замене уравнения $v=1$ формулой $a=1 \vee \Theta(\gamma)$. Более точные определения и формулировку основного результата приведем далее. Можно сказать, что в отличие от классической теоремы Гильберта показатель степени $\gamma$ зависит от переменных.

Отметим, что наши доказательства существенно опираются на упомянутый результат о нётеровости по уравнениям жестких групп из [4], описание координатных групп неприводимых алгебраических множеств над делимой жесткой группой из [7] и на технику, развитую в этих работах.

Заметим, что в работе [2] (параграф "Радикалы и обобщенная Nullstellensatz") был предложен общий подход к построению логических следствий (радикалов в $G$-группах) на языке формальных логических систем. При этом подходе можно обходиться без алгебраически замкнутых объектов, поскольку решения уравнений рассматриваются во всех $G$-группах данного класса. В частности, было показано, что если группа $G$ является нётеровой по уравнениям, то логические следствия относительно $G$ полностью определяются квазимногообразием, порожденным группой $G$ (квазиэквациональной теорией группы $G$ ), что дает способ построения радикалов. Однако в силу своей общности данное описание радикалов трудно применять на практике, особенно в тех классах групп, где квазиэквациональная теория сложна или недостаточно изучена. Наша точка зрения состоит в том, что, прежде чем доказать теорему Гильберта о нулях в хорошей формулировке, необходимо подробно изучить алгебраическую геометрию над (алгебраически замкнутыми) группами данного класса, что было уже сделано в случае жестких групп.

Автор благодарит А.Г. Мясникова за полезные обсуждения тематики работы.

\section{§ 2. Некоторые определения и известные факты}

2.1. Как обычно, для элементов $a, b$ данной группы полагаем

$$
a^{b}=b^{-1} a b, \quad[a, b]=a^{-1} b^{-1} a b .
$$

Напомним, что если $A$ - абелева нормальная подгруппа в группе $G$, то действие $G$ на $A$ сопряжениями определяет действие целочисленного группового кольца $\mathbb{Z}[G / A]$, вследствие чего на $A$ возникает структура правого $\mathbb{Z}[G / A]$-модуля.

2.2. Группа $G$ называется $m$-жесткой, если в ней существует нормальный ряд

$$
G=G_{1}>G_{2}>\cdots>G_{m}>G_{m+1}=1,
$$

факторгруппы $G_{i} / G_{i+1}$ которого абелевы и как правые $\mathbb{Z}\left[G / G_{i}\right]$-модули не имеют модульного кручения. Из [8] следует, что такой ряд, если он вообще существует, определяется группой $G$ однозначно, кроме того, ступень разрешимости группы в точности равна $m$. Назовем соответствующий ряд жестким и 
для его членов введем обозначения $G_{i}=\rho_{i}(G)$. Жесткой группой называется $m$-жесткая для некоторого $m$ группа. Важными примерами жестких групп являются свободные разрешимые группы. Класс жестких групп замкнут относительно подгрупп и факторгрупп вида $G / \rho_{i}(G)$. Целочисленное групповое кольцо $\mathbb{Z} G$ жесткой группы $G$ является кольцом Оре, через $Q(G)$ обозначим его тело частных.

Согласно [5] жесткая группа $G$ называется делимой, если элементы факторгруппы $\rho_{i}(G) / \rho_{i+1}(G)$ делятся на ненулевые элементы кольца $\mathbb{Z}\left[G / \rho_{i}(G)\right]$, или, другими словами, $\rho_{i}(G) / \rho_{i+1}(G)$ является (правым) векторным пространством над телом частных $Q\left(G / \rho_{i}(G)\right)$ этого кольца. Наконец, жесткая группа $G$ называется расщепимой, если она распадается в последовательное полупрямое произведение $A_{1} A_{2} \cdots A_{m}$ ( $A_{i}$ нормализует $A_{j}$ при $i<j$ ) абелевых групп $A_{i} \cong \rho_{i}(G) / \rho_{i+1}(G)$ и $A_{i} A_{i+1} \cdots A_{m}=\rho_{i}(G)$. Делимая расщепимая жесткая группа определяется однозначно с точностью до изоморфизма мощностями $\alpha_{i}$ баз соответствующих векторных пространств $A_{i}$, она обозначается через $M\left(\alpha_{1}, \ldots, \alpha_{m}\right)$. В [6] доказано, что любая делимая группа расщепляется, т. е. изоморфна группе вида $M\left(\alpha_{1}, \ldots, \alpha_{m}\right)$. Говорят, что $m$-жесткая группа $G$ вложена в $m$-жесткую группу $H$ с сохранением линейной независимости, если любая система элементов из $\rho_{i}(G) / \rho_{i+1}(G)$, линейно независимая над кольцом $\mathbb{Z}\left[G / \rho_{i}(G)\right]$, остается линейно независимой и над кольцом $\mathbb{Z}\left[H / \rho_{i}(H)\right]$. Здесь используются равенства $\rho_{i}(G)=\rho_{i}(H) \cap G$, вследствие чего можно считать

$$
\rho_{i}(G) / \rho_{i+1}(G) \leqslant \rho_{i}(H) / \rho_{i+1}(H), \quad \mathbb{Z}\left[G / \rho_{i}(G)\right] \leqslant \mathbb{Z}\left[H / \rho_{i}(H)\right] .
$$

В [5] установлено, что всякая $m$-жесткая группа вкладывается с сохранением линейной независимости в подходящую группу $M\left(\alpha_{1}, \ldots, \alpha_{m}\right)$. Вложения с сохранением линейной независимости для краткости назовем независимыми, они играют важную роль в теории жестких групп.

Обозначим через $\Sigma_{m}$ класс всех жестких групп ступени разрешимости $\leqslant m$. Будем говорить, что нетривиальная группа $G \in \Sigma_{m}$ алгебраически замкнута в классе $\Sigma_{m}$ (по независимым вложениям), если из того, что $G$ независимо вложена в $H \in \Sigma_{m}$, следует, что любая система уравнений от конечного числа переменных с коэффициентами из $G$, имеющая решение над $H$, имеет решение над $G$. Соответственно группа $G$ называется экзистенциально замкнутой (по независимым вложениям), если любая $\exists$-формула с коэффициентами из $G$, истинная на $H$, истинна на $G$. В [6] доказано, что алгебраически замкнутые в классе $\Sigma_{m}$ группы - это в точности делимые $m$-жесткие группы, они же являются в точности экзистенциально замкнутыми объектами в рассматриваемом классе.

2.3. Итак, мы будем рассматривать делимую $m$-жесткую группу $G=$ $M\left(\alpha_{1}, \ldots, \alpha_{m}\right)$. Зафиксируем ее расщепление $A_{1} A_{2} \cdots A_{m}$ в полупрямое произведение абелевых подгрупп. Полагаем для краткости $A=A_{1} \cdots A_{m-1}$. Группу $G$ можно отождествить с группой матриц $\left(\begin{array}{ll}A & 0 \\ T & 1\end{array}\right)=A A_{m}$, где $T=$ $t_{1} Q(A)+\cdots-$ векторное пространство над телом $Q(A)$ с базой $\left\{t_{1}, \ldots\right\}$ мощности $\alpha_{m}, A_{m}=\left(\begin{array}{cc}1 & 0 \\ T & 1\end{array}\right)$. 
Важный момент состоит в следующем. Как и в [4], мы обычные переменные $x_{1}, \ldots, x_{n}$ расщепим, т. е. представим каждое $x_{i}$ в виде произведения $x_{i 1} \cdots x_{i m}$, где $x_{i j}$ принимает значение в $A_{j}$. Новые переменные назовем специалъными. На самом деле специальные переменные можно рассматривать как обычные, но при этом они должны удовлетворять уравнениям $\left[x_{i j}, a_{j}\right]=1$, где $a_{j}$ - какой-то нетривиальный элемент из $A_{j}$. Пусть $X$ обозначает множество всех специальных переменных, $X^{\prime}$ - его подмножество, состоящее из переменных $x_{i j}$ с условием $j<m$. Естественным образом определяются спещиальные алгебраические множества, они будут подмножествами множества $\left(A_{1} \times A_{2} \times \cdots \times A_{m}\right)^{n}$, которое можно отождествить с $G^{n}$. Также естественно определяются координатные группы специальных алгебраических множеств.

В работе [4] построена координатная группа $D=G[X]$ аффинного пространства $G^{n}$ в специальных переменных $X$. Укажем ее вид. Пусть по индукции построена координатная группа $C=A\left[X^{\prime}\right]$ аффинного пространства $A^{n}$ в специальных переменных $X^{\prime}$, она является $(m-1)$-жесткой и расщепляется в полупрямое произведение $D_{1} \cdots D_{m-1}$ абелевых подгрупп. Рассмотрим правый $\mathbb{Z} C$-модуль $T_{C}$, который является прямой суммой модуля $T \otimes_{\mathbb{Z} A} \mathbb{Z} C$ и свободного модуля $x_{1 m} \cdot \mathbb{Z} C+\cdots+x_{n m} \cdot \mathbb{Z} C$ с базой $\left\{x_{1 m}, \ldots, x_{n m}\right\}$. Тогда полагаем

$$
D=G[X]=\Gamma\left(G^{n}\right)=\left(\begin{array}{cc}
C & 0 \\
T_{C} & 1
\end{array}\right)=D_{1} \cdots D_{m}=C D_{m},
$$

где $D_{m}=\left(\begin{array}{rr}1 & 0 \\ T_{C} & 1\end{array}\right)$. При этом переменная $x_{i j}$ для $j<m$ отождествляется с матрицей $\left(\begin{array}{cc}x_{i j} & 0 \\ 0 & 1\end{array}\right)$, а для $j=m-$ с матрицей $\left(\begin{array}{cc}1 & 0 \\ x_{i m} & 1\end{array}\right)$. Имеется канонический гомоморфизм модуля $T \otimes_{\mathbb{Z} A} \mathbb{Z} C$ в векторное пространство $t_{1} \cdot Q(C)+\cdots$ над телом $Q(C)$ с базой $\left\{t_{1}, \ldots\right\}$. Из [7, лемма 3] следует, что этот гомоморфизм является вложением; в частности, модуль $T \otimes_{\mathbb{Z} A} \mathbb{Z} C$ не имеет кручения.

Отметим, что если $f \in D$ и $f=f_{1} \cdots f_{m}$, где $f_{j} \in D_{j}$, то при любых значениях специальных переменных $X$ значения $f_{j}$ принадлежат $A_{j}$. В частности, если для данных значений переменных $f=1$, то и все $f_{j}=1$.

2.4. Нам понадобится рассматривать так называемые кольщевые уравнения. В нашей ситуации это будут уравнения вида $\gamma=0$, где $\gamma \in \mathbb{Z} C$, при этом значения переменных $x_{i j}$ по-прежнему ищутся в $A_{j}$. Понятно, что если некоторые значения $x_{i j} \in A_{j}$ удовлетворяют уравнению, то $\gamma \in(C-1) \mathbb{Z} C$. Во введении мы определили позитивную формулу $\Theta(\gamma)$, в нашем случае она зависит от переменных $X^{\prime}$. Легко видеть, что эта формула логически равносильна уравнению $\gamma=0$.

\section{§ 3. Алгебраические следствия и формулировка теоремы}

Позитивной формулой от $X$ над $G$ будем называть булеву комбинацию без отрицаний уравнений от $X$ над $G$ либо утверждений “истина" или “ложь”. Если $g$ - нетривиальный элемент группы $G$, то утверждение $g=1$ считаем ложью, утверждение $1=1$ является истиной. Обычно позитивную формулу будем представлять в дизъюнктивной нормальной форме $\Phi=\Phi_{1} \vee \cdots \vee \Phi_{k}$. 
Назовем стандартными правилами вывода для позитивных формул следующие два типа:

1) правила вывода

$$
\begin{gathered}
(f=1) \vdash\left(f^{-1}=1\right), \quad(f=1 \wedge h=1) \vdash(f h=1), \quad(f=1) \vdash\left(f^{h}=1\right), \\
f, h \in D,
\end{gathered}
$$

которые соответствуют построению нормальной подгруппы, порожденной данными уравнениями;

2) пропозициональные правила вывода, взятые из исчисления высказываний и аксиом булевой алгебры без отрицаний.

Также будут применяться два дополнительных правила вывода.

3) Расщепление уравнения. Пусть $f \in D$ и $f=f_{1} \cdots f_{m}$, где $f_{j} \in D_{j}$. Правило вывода определяется в виде

$$
(f=1) \vdash\left(f_{1}=1 \wedge \cdots \wedge f_{m}=1\right) .
$$

С учетом этого правила можно считать, что всякое уравнение вида $f=1$ переписывается как система $f_{1}=1 \wedge \cdots \wedge f_{m}=1$.

4) Разветвление уравнения. По индукции предполагаем, что это правило уже определено для уравнений $f=1$ при $f \in D_{1} \cup D_{2} \cup \cdots \cup D_{m-1}$. Пусть $f=$ $\left(\begin{array}{ll}1 & 0 \\ v & 1\end{array}\right) \in D_{m}=\left(\begin{array}{cc}1 & 0 \\ T_{C} & 1\end{array}\right)$. Предположим, элемент $v \in V$ делится в $T_{C}$ на $\gamma \in \mathbb{Z} C$, т. е. $v=u \gamma$, где $u \in T_{C}$. Полагаем $h=\left(\begin{array}{ll}1 & 0 \\ u & 1\end{array}\right)$. Понятно, что уравнение $f=1$, которое переписывается в виде $v=0$, логически эквивалентно дизъюнкции $u=0 \vee \gamma=0$. В свою очередь, мы знаем, что кольцевое уравнение $\gamma=0$ эквивалентно позитивной формуле $\Theta(\gamma)$, которая была определена во введении. Правило вывода определяется следующим образом:

$$
(f=1) \vdash(f=1) \wedge((h=1) \vee \Theta(\gamma)) .
$$

Данная позитивная формула $\Phi=\Phi_{1} \vee \cdots \vee \Phi_{k}$ задает в аффинном пространстве $G^{n}$ замкнутое подмножество, которое обозначим через $S(\Phi)$. Оно является объединением алгебраических множеств $S\left(\Phi_{1}\right), \ldots, S\left(\Phi_{k}\right)$. Назовем $S(\Phi)$ опорным множеством формулы $\Phi$.

Назовем верхней частъю формуль $\Phi=\Phi_{1} \vee \cdots \vee \Phi_{k}$ с расщепленными уравнениями подформулу $\Phi^{\prime}=\Phi_{1}^{\prime} \vee \cdots \vee \Phi_{k}^{\prime}$, в которой оставлены только уравнения от $X^{\prime}$ над $A$, т.е. уравнения, левые части которых принадлежат $D_{1} \cup D_{2} \cup \cdots \cup D_{m-1}$. Обозначим через $S^{\prime}(\Phi)$ опорное множество формулы $\Phi^{\prime}$ в $A^{n}$. Назовем $S^{\prime}(\Phi)$ верхним множеством формулы $\Phi$. Понятно, что формула $\Phi^{\prime}$ является пропозициональным следствием формулы $\Phi$ (получается применением пропозициональных правил вывода) и множество $S^{\prime}(\Phi)$ содержит проекцию множества $S(\Phi)$ на $A^{n}$. Отметим, что если формула $\Psi$ получается из $\Phi$ с помощью разветвления уравнения, то $S(\Phi)=S(\Psi), S^{\prime}(\Phi) \supseteq S^{\prime}(\Psi)$ и $\Phi$ является логическим следствием $\Psi$.

Нас будут интересовать следствия данной формулы $\Phi$ во множестве позитивных формул. При этом будем рассматривать логические следствия и алгебраические следствия. По определению формула $\Psi$ является логическим следствием формулы $\Phi$, если $S(\Psi) \supseteq S(\Phi)$. Формулы, получаемые из $\Phi$ с помощью 
стандартных правил вывода, расщеплений и разветвлений уравнений, назовем алгебраическими следствиями Ф. Из определений следует, что множество алгебраических следствий содержится во множестве логических следствий.

Сформулируем основной результат.

Теорема. Для любой позитивной формуль от $X$ над $G=M\left(\alpha_{1}, \ldots, \alpha_{m}\right)$ множество ее алгебраических следствий совпадает с множеством логических следствий.

Отсюда получается и слабая форма теоремы Гильберта: формула $\Phi$ не имеет решений в $G^{n}$ тогда и только тогда, когда среди ее алгебраических следствий содержится ложь.

ЗАМЕчАНИЕ 1. Правило вывода разветвления уравнений

$$
(f=1) \vdash(f=1) \wedge((h=1) \vee \Theta(\gamma))
$$

можно было бы заменить правилом

$$
(f=1) \vdash(h=1) \vee \Theta(\gamma),
$$

так как формулы $(f=1) \wedge((h=1) \vee \Theta(\gamma))$ и $(h=1) \vee \Theta(\gamma)$ эквивалентны по стандартным правилам вывода.

ЗАмЕчАНИЕ 2. При определении алгебраических следствий мы можем исключить из правил вывода расщепления уравнений, поскольку они получаются из остальных правил вывода.

Действительно, замечание 2 справедливо. Пусть $f=\left(\begin{array}{ll}c & 0 \\ v & 1\end{array}\right) \in D, c \in C$, $v \in T_{C}$. Выберем нетривиальный элемент $g=\left(\begin{array}{cc}1 & 0 \\ t & 1\end{array}\right) \in G$ и рассмотрим коммутатор $[f, g]=\left(\begin{array}{cc}1 & 0 \\ t(1-c) & 1\end{array}\right)$. Уравнение $[f, g]=1$ получается из $f=1$ с помощью стандартных правил вывода. Из него с помощью разветвления получаем уравнение $c=1$. Далее по индукции можно считать, что последнее уравнение расщепляется с помощью стандартных правил вывода и разветвлений. Отсюда следует, что это верно и для уравнения $f=1$.

\section{§ 4. Доказательство теоремы}

По индукции предполагаем, что утверждение теоремы выполнено для формул от $X^{\prime}$ над $A$. Также предполагаем, что в формулах все уравнения расщеплены.

4.1. Нам понадобятся некоторые конструкции и две вспомогательные леммы.

Пусть в этом пункте формула $\Phi$ является системой уравнений от $X$ над $G$ и множество $S^{\prime}=S^{\prime}(\Phi)$ непусто и неприводимо. Пусть $\bar{C}=\Gamma\left(S^{\prime}\right)=C / I\left(S^{\prime}\right)-$ координатная группа множества $S^{\prime}$. Как показано в [7], группа $\bar{C}$ будет $(m-1)$ жесткой, поэтому кольцо $\mathbb{Z} \bar{C}$ является кольцом Оре, модуль $T \otimes_{\mathbb{Z} A} \mathbb{Z} \bar{C}$ над этим 
кольцом вкладывается каноническим образом в векторное пространство над телом $Q(\bar{C})$. Полагаем

$$
\begin{aligned}
& T_{\bar{C}}=T \otimes_{\mathbb{Z} A} \mathbb{Z} \bar{C}+x_{1 m} \cdot \mathbb{Z} \bar{C}+\cdots+x_{n m} \cdot \mathbb{Z} \bar{C} \\
& \bar{D}=\left(\begin{array}{cc}
\bar{C} & 0 \\
T_{\bar{C}} & 1
\end{array}\right)=\bar{C} \cdot \bar{D}_{m}, \quad \bar{D}_{m}=\left(\begin{array}{cc}
1 & 0 \\
T_{\bar{C}} & 1
\end{array}\right) .
\end{aligned}
$$

Имеются канонические эпиморфизмы групп $C \rightarrow \bar{C}$, колец $\mathbb{Z} C \rightarrow \mathbb{Z} \bar{C}$, модулей $T_{C} \rightarrow T_{\bar{C}}$ и групп $D \rightarrow \bar{D}$. Чертой сверху обозначаем применение соответствующего эпиморфизма. Рассмотрим нормальную подгруппу $H$ из $D$, порожденную левыми частями уравнений формулы $\Phi$. Она разлагается в полупрямое произведение: $H=H^{\prime} H_{m}$, где $H^{\prime} \triangleleft C$,

$$
H_{m}=\left(\begin{array}{cc}
1 & 0 \\
V & 1
\end{array}\right) \leqslant D_{m}=\left(\begin{array}{cc}
1 & 0 \\
T_{C} & 1
\end{array}\right) .
$$

Множество $V$ является подмодулем в $T_{C}$, а $\bar{V}$ - подмодулем в $T_{\bar{C}}$. Обозначим через $W$ изолятор подмодуля $\bar{V}$ в $T \bar{C}$.

Лемма 1. Пусть $u \in T_{C} u \bar{u} \in W$. Пусть $\bar{u} \cdot \bar{\gamma} \in \bar{V}$, где $0 \neq \bar{\gamma} \in \mathbb{Z} \bar{C}$. Тогда к системе уравнений $\Phi$ можно добавить несколько уравнений от $X^{\prime}$ над $A$, которые являются алгебраическими следствиями $\Phi^{\prime}$, так что в новой ситуации иү будет принадлежать $V$.

ДоказАтельство. По условию $\bar{u} \cdot \bar{\gamma}=\bar{v} \in \bar{V}$ для некоторого $v \in V$. Тогда $v-u \gamma$ лежит в ядре гомоморфизма $T_{C} \rightarrow T_{\bar{C}}$. Это ядро как подмодуль в $T_{C}$ порождается элементами вида $z(f-1)$, где $z \in T_{C}, f \in I\left(S^{\prime}\right)$. Выберем конечное множество $\left\{z_{1}\left(f_{1}-1\right), \ldots, z_{r}\left(f_{r}-1\right)\right\}$ элементов такого вида, через которое выражается $v-u \gamma$. Добавим к $\Phi$ систему уравнений $f_{1}=1 \wedge \cdots \wedge f_{r}=1$, при этом $H^{\prime}$ заменится на подгруппу $\left\langle H^{\prime}, f_{1}, \ldots, f_{r}\right\rangle$. В новой ситуации $u \gamma$ будет принадлежать $V$. Осталось отметить, что любое уравнение $f=1$, где $f \in I\left(S^{\prime}\right)$, является логическим следствием системы $\Phi^{\prime}$, а значит, по индукции и алгебраическим следствием. Лемма доказана.

Лемма 2. Если $W \cap T=0$, то опорное множество формуль $\Phi$ непусто.

ДоказАтельство. Рассмотрим группу $\left(\begin{array}{cc}\bar{C} & 0 \\ T_{\bar{C}} / W & 1\end{array}\right)$. Согласно [7, теорема 3] она является координатной группой некоторого неприводимого алгебраического множества $S \subseteq G^{n}$, причем замыкание проекции $S$ на $A^{n}$ равно $S^{\prime}$, так что $S$ непусто. Из построений вытекает, что формула $\Phi$ выполняется на $S$, т. е. $S(\Phi) \supseteq S$. Лемма доказана.

4.2. Сначала докажем аналог теоремы Гильберта о нулях в слабой формулировке.

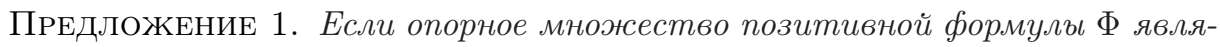
ется пустым, то среди алгебраических следствий $\Phi$ содержится ложь. 
ДоказАтельство. Достаточно рассмотреть случай, когда $\Phi$ является системой уравнений. Пусть $S^{\prime}=S^{\prime}(\Phi)$. Если множество $S^{\prime}$ пусто, то по индукционному предположению среди алгебраических следствий формулы $\Phi^{\prime}$, а значит, и $\Phi$ имеется ложь. Допустим, что $S^{\prime}$ непусто. Используя нётеровость топологии Зарисского, по индукции предполагаем, что предложение выполняется для любой системы уравнений $\Psi$ с условием $S(\Psi)=\varnothing$ и $S^{\prime}(\Psi) \varsubsetneqq S^{\prime}$.

Разберем сначала случай, когда множество $S^{\prime}$ приводимо. Пусть $S^{\prime}=$ $S_{1}^{\prime} \cup \cdots \cup S_{k}^{\prime}$ - разложение на неприводимые компоненты. Выберем элементы $f_{1}, \ldots, f_{k} \in C$ такие, что $f_{i} \in I\left(S_{i}^{\prime}\right) \backslash I\left(S^{\prime}\right)$. Формула $f_{1}=1 \vee \cdots \vee f_{k}=1$ является логическим следствием формулы $\Phi^{\prime}$, а значит, по индукции и алгебраическим следствием. Тогда $\Phi \wedge\left(f_{1}=1\right) \vee \cdots \vee \Phi \wedge\left(f_{k}=1\right)$ будет алгебраическим следствием формулы $\Phi$. Поскольку $S^{\prime}\left(\Phi \wedge\left(f_{i}=1\right)\right) \varsubsetneqq S^{\prime}$, можно утверждать, что каждая компонента $\Phi \wedge\left(f_{i}=1\right)$ среди алгебраических следствий содержит ложь. Отсюда следует аналогичный вывод для $\Phi$.

Предположим, что $S^{\prime}$ - неприводимое алгебраическое множество. Будем использовать конструкции и обозначения из п 3.1. Из леммы 2 следует, что $W \cap T>0$. Пусть $0 \neq t \in W \cap T$. Тогда существует ненулевой элемент $\bar{\gamma} \in \mathbb{Z} \bar{C}$ такой, что $t \bar{\gamma}=\bar{v} \in \bar{V}, v \in V$. По лемме $1 \mathrm{k} \Phi$ можно добавить несколько уравнений, которые являются алгебраическими следствиями $\Phi^{\prime}$, так что в новой ситуации имеем $t \gamma \in V$. Теперь рассмотрим разветвление

$$
\Phi \rightarrow \Phi \wedge(t=0) \vee \Phi \wedge \Psi_{1} \vee \cdots \vee \Phi \wedge \Psi_{r}
$$

где $\Psi_{1} \vee \cdots \vee \Psi_{r}$ - дизъюнкция уравнений от $X^{\prime}$ над $A$, равносильная $\gamma=0$. Формула $t=0$ является ложью. Отметим также, что для каждого $i$ множество $S^{\prime}\left(\Phi \wedge \Psi_{i}\right)$ строго меньше $S^{\prime}$, следовательно, по индукции среди алгебраических следствий каждой формулы $\Phi \wedge \Psi_{i}$, а значит, и формулы $\Phi$ есть ложь. Предложение доказано.

4.3. Назовем элементарными преобразованиями формулы $\Phi$ разветвления или умножения (имеется в виду операция конъюнкции) какой-либо дизъюнктивной компоненты $\Phi_{i}$ на формулу от $X^{\prime}$ над $A$, которая являются следствием $\Phi_{i}^{\prime}$. Из определений следует, что если одна формула получается из другой элементарными преобразованиями, то эти формулы алгебраически эквивалентны.

Пусть $\Phi$ является системой уравнений от $X$ над $G, S=S(\Phi), S^{\prime}=$ $S^{\prime}(\Phi)$. Предположим, что формула $\Psi_{1} \vee \cdots \vee \Psi_{r}$ получается из $\Phi$ при помощи элементарных преобразований. Тогда можно утверждать, что $S\left(\Psi_{i}\right) \subseteq S$, $S^{\prime}\left(\Psi_{i}\right) \subseteq S^{\prime}$. Назовем систему уравнений $\Phi$ пределъной, если для любой формулы $\Psi_{1} \vee \cdots \vee \Psi_{r}$, полученной из $\Phi$ при помощи элементарных преобразований, выполняются условия:

1) хотя бы одно множество $S\left(\Psi_{i}\right)$ равно $S$;

2) если $S\left(\Psi_{i}\right)=S$, то $S^{\prime}\left(\Psi_{i}\right)=S^{\prime}$.

Произвольную позитивную формулу назовем предельной, если предельными являются все ее дизъюнктивные компоненты. 
ПрЕДЛОжЕНИЕ 2. Любую позитивную формулу с помощъю элементарных преобразований можно превратить в предельную.

ДокАЗАтЕльство. Пусть формула $\Phi$ является контрпримером. Тогда одна из ее дизъюнктивных компонент (пусть $\Phi_{1}$ ) также является контрпримером. По предположению из $\Phi_{1}$ путем элементарных преобразований можно получить формулу, каждая дизьюнктивная компонента которой не удовлетворяет либо условию 1), либо условию 2). Одна из этих компонент (пусть $\Phi_{2}$ ) также должна быть контрпримером и т.д. Получаем бесконечную последовательность формул $\Phi_{1}, \Phi_{2}, \ldots$ такую, что для любого индекса выполнены включения $S\left(\Phi_{i}\right) \supseteq S\left(\Phi_{i+1}\right), S^{\prime}\left(\Phi_{i}\right) \supseteq S^{\prime}\left(\Phi_{i+1}\right)$ и хотя бы одно из двух включений строгое. Это противоречит нётеровости топологии Зарисского на $G^{n}$ или $A^{n}$. Предложение доказано.

ПредложЕниЕ 3. Пусть система уравнений $\Phi$ является предельной и ее опорное множество $S=S(\Phi)$ непусто. Тогда оно неприводимо и замыканием проекции $S$ на $A^{n}$ является $S^{\prime}=S^{\prime}(\Phi)$.

ДокАЗАТЕЛЬство. Предположим сначала, что множество $S^{\prime}$ приводимо и $S^{\prime}=S_{1}^{\prime} \cup \cdots \cup S_{k}^{\prime}-$ разложение на неприводимые компоненты. Выберем элементы $f_{1}, \ldots, f_{k} \in C$ такие, что $f_{i} \in I\left(S_{i}^{\prime}\right) \backslash I\left(S^{\prime}\right)$. Формула $f_{1}=1 \vee \cdots \vee f_{k}=1$ является следствием формулы $\Phi^{\prime}$. Формула $\Phi \wedge\left(f_{1}=1\right) \vee \cdots \vee \Phi \wedge\left(f_{k}=1\right)$, полученная из $\Phi$ с помощью элементарного преобразования, не удовлетворяет условию 2). Это противоречит предельности $\Phi$.

Итак, множество $S^{\prime}$ неприводимо. Далее используем конструкции и обозначения из п. 4.1. Покажем, что $W \cap T=0$. Допустим противное, а именно $W \cap T>0$ и $0 \neq t \in W \cap T, 0 \neq \bar{\gamma} \in \mathbb{Z} \bar{C}, t \bar{\gamma}=\bar{v} \in \bar{V}, v \in V$. По лемме 1 к $\Phi$ можно добавить несколько уравнений, которые являются алгебраическими следствиями $\Phi^{\prime}$ так, что в новой ситуации $t \gamma \in V$. Теперь рассмотрим разветвление

$$
\Phi \rightarrow \Phi \wedge(t=0) \vee \Phi \wedge \Psi_{1} \vee \cdots \vee \Phi \wedge \Psi_{r}
$$

где $\Psi_{1} \vee \cdots \vee \Psi_{r}$ - дизъюнкция уравнений от $X^{\prime}$ над $A$, равносильная $\gamma=0$. Снова компоненты разложения не удовлетворяет условию 1) или 2), что противоречит предельности $\Phi$.

Рассмотрим теперь группу $\left(\begin{array}{cc}\bar{C} & 0 \\ T_{\bar{C}} / W & 1\end{array}\right)$. Согласно [7, теорема 3] она является координатной группой некоторого неприводимого алгебраического множества $P \subseteq G^{n}$, причем замыкание проекции $P$ на $A^{n}$ равно $S^{\prime}$. Покажем, что $P=S$. По построению $P \subseteq S$, так как формула $\Phi$ на $P$ выполняется. Для доказательства обратного включения необходимо установить, что если $f \in I(P)$, то $f(S)=1$. Пусть $f=f^{\prime} f_{m}$, где $f^{\prime} \in C, f_{m}=\left(\begin{array}{ll}1 & 0 \\ u & 1\end{array}\right) \in D_{m}$. Поскольку $f^{\prime}(S)=f^{\prime}\left(S^{\prime}\right)=1$, достаточно доказать, что $u(S)=0$. Известно, что $\bar{u} \in W$. Пусть $0 \neq \bar{\gamma} \in \mathbb{Z} \bar{C}, \bar{u} \cdot \bar{\gamma}=\bar{v} \in \bar{V}, v \in V$. С использованием леммы 1 можно предполагать, что $u \gamma \in V$. Берем разветвление $\Phi \rightarrow \Phi \wedge(u=0) \vee \Phi \wedge(\gamma=0)$. Из условия предложения следует, что опорное множество любой дизъюнктивной 
компоненты формулы $\Phi \wedge(\gamma=0)$ должно быть меньше $S$, иначе не выполняется 2). Тогда опорное множество формулы $\Phi \wedge(u=0)$ должно быть равно $S$. Отсюда $u(S)=0$. Предложение доказано.

ПрЕДЛОЖЕНИЕ 4. Пусть система уравнений $\Phi$ является пределъной и ее опорное множество $S=S(\Phi)$ непусто. Тогда любое логичекое следствие формуль $\Phi$ является ее алгебраическим следствием.

ДоКАЗАТЕЛЬСтво. Пусть $S^{\prime}=S^{\prime}(\Phi)$. Предположим, что формула $\Psi=$ $\Psi_{1} \vee \cdots \vee \Psi_{r}$ является логическим следствием $\Phi$. Тогда $S(\Psi) \supseteq S$. Поскольку последнее множество неприводимо, некоторое $S\left(\Psi_{i}\right)$ содержит $S$. Поэтому можно считать, что $\Psi$ является системой уравнений. Достаточно установить, что любое уравнение $f=1$, где $f \in I(S)$, является алгебраическим следствием $\Phi$. Пусть $f=f^{\prime} f_{m}$, где $f^{\prime} \in C, f_{m}=\left(\begin{array}{ll}1 & 0 \\ u & 1\end{array}\right) \in D_{m}$. Поскольку $f^{\prime}\left(S^{\prime}\right)=1$, по индукции формула $f^{\prime}=1$ является алгебраическим следствием формулы $\Phi^{\prime}$, которая в свою очередь является алгебраическим следствием формулы $\Phi$. Поэтому задача сводится к случаю, когда $f=f_{m}$. С учетом нётеровости топологии Зарисского на $G^{n}$ можно по индукции предполагать, что утверждение предложения верно для формул, удовлетворяющих его условию, опорное множество которых меньше $S$. Поскольку $u(S)=0$, в обозначениях из п. 4.1 имеем $\bar{u} \in W$. Пусть $0 \neq \bar{\gamma} \in \mathbb{Z} \bar{C}, \bar{u} \cdot \bar{\gamma}=\bar{v} \in \bar{V}, v \in V$. С использованием леммы 1 можно предполагать, что $u \gamma \in V$. Рассмотрим разветвление

$$
\Phi \rightarrow \Phi \wedge(u=0) \vee \Phi \wedge(\gamma=0)=\Phi \wedge(u=0) \vee \Phi \wedge \Phi_{1} \vee \cdots \vee \Phi \wedge \Phi_{k}
$$

здесь $\gamma=0$ переписывается в виде $\Phi_{1} \vee \cdots \vee \Phi_{k}$. Поскольку формула $\Phi$ предельна и множества $S^{\prime}\left(\Phi \wedge \Phi_{i}\right)$ строго меньше $S^{\prime}$, множества $S\left(\Phi \wedge \Phi_{i}\right)$ должны быть строго меньше $S$. Тогда по индукции формула $u=0$ является алгебраическим следствием каждой формулы $\Phi \wedge \Phi_{1}, \ldots, \Phi \wedge \Phi_{k}$. Значит, $u=0$ является алгебраическим следствием $\Phi \wedge(u=0) \vee \Phi \wedge \Phi_{1} \vee \cdots \vee \Phi \wedge \Phi_{k}$, а потому и $\Phi$. Предложение доказано.

4.4. Наконец, мы можем завершить доказательство теоремы. Пусть формула $\Psi=\Psi_{1} \vee \cdots \vee \Psi_{r}$ является логическим следствием формулы $\Phi$, т. е. $S(\Psi) \supseteq$ $S(\Phi)$. Если $S(\Phi)=\varnothing$, то применяем предложение 1. Пусть множество $S(\Phi)$ непусто. По предложению 2 формулу $\Phi$ можно заменить предельной. Затем оставим в этой формуле только те дизъюнктивные компоненты, которые соответствуют разложению множества $S(\Phi)$ на неприводимые подмножества, так как по предложениям 3, 4 каждая выброшенная дизъюнктивная компонента будет давать в качестве алгебраического следствия одну из оставленных, а потому новая формула будет алгебраическим следствием старой. Пусть в результате $\Phi=\Phi_{1} \vee \cdots \vee \Phi_{k}$. Мы можем утверждать, что найдутся $\Psi_{i_{1}}, \ldots, \Psi_{i_{k}}$ такие, что $S\left(\Phi_{1}\right) \subseteq S\left(\Psi_{i_{1}}\right), \ldots, S\left(\Phi_{k}\right) \subseteq S\left(\Psi_{i_{k}}\right)$. Тогда по предложению 4 формула 
$\Psi_{i_{1}} \vee \cdots \vee \Psi_{i_{k}}$, а значит, и формула $\Psi$ являются алгебраическим следствием формулы $\Phi$. Теорема доказана.

\section{Список литературы}

1. G. Baumslag, A. Myasnikov, V. Remeslennikov, "Algebraic geometry over groups. I. Algebraic sets and ideal theory", J. Algebra, 219:1 (1999), 16-79.

2. A. Myasnikov, V. Remeslennikov, "Algebraic geometry over groups. II. Logical foundations", J. Algebra, 234:1 (2000), 225-276.

3. W.R. Scott, "Algebraically closed groups", Proc. Amer. Math. Soc., 2:1 (1951), 118-121.

4. Н. С. Романовский, "Нетеровость по уравнениям жестких разрешимых групп", Алгебра и логика, 48:2 (2009), 258-279; англ. пер.: N. S. Romanovskii, "Equational Noetherianness of rigid soluble groups", Algebra Logic, 48:2 (2009), 147-160.

5. Н. С. Романовский, “Делимые жесткие группы”, Алгебра и логика, 47:6 (2008), 762-776; англ. пер.: N. S. Romanovskii, "Divisible rigid groups", Algebra Logic, 47:6 (2008), 426-434.

6. А. Г. Мясников, Н. С. Романовский, "Логические аспекты теории делимых жестких групп”, Докл. РАН, 459:2 (2014), 154-155; англ. пер.: А. G. Myasnikov, N. S. Romanovskii, "Logical aspects of the theory of divisible rigid groups", Dokl. Math., 90:3 (2014), 697-698.

7. Н. С. Романовский, "Неприводимые алгебраические множества над делимыми распавшимися жесткими группами", Алгебра и логика, 48:6 (2009), 793-818; англ. пер.: N.S. Romanovskii, "Irreducible algebraic sets over divisible decomposed rigid groups", Algebra Logic, 48:6 (2009), 449-464.

8. A. Myasnikov, N. Romanovskiy, "Krull dimension of solvable groups", J. Algebra, 324:10 (2010), 2814-2831.

НикоЛАЙ СЕМЕНОВИч РОМАНОВСКИЙ

(Nikolai S. Romanovskiy)

Институт математики им. С.Л. Соболева

Сибирского отделения Российской академии наук,

г. Новосибирск

E-mail: rmnvski@math.nsc.ru
Поступило в редакцию 10.09.2014 